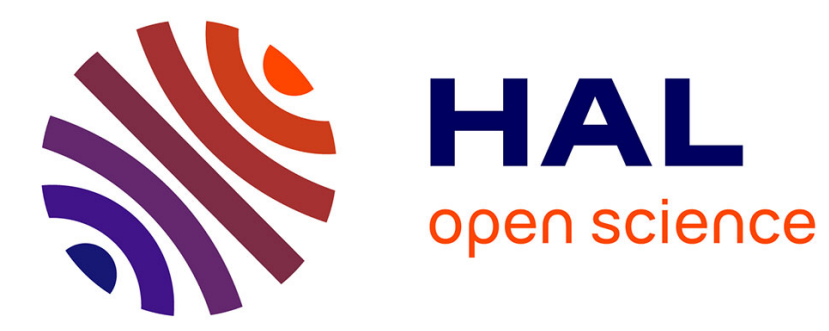

\title{
Estimation des flux de matières en suspension lors des chasses hydrauliques de l'Arc de 2006 À 2011
}

\author{
G. Antoine, M. Jodeau, B. Camenen, M. Esteves, J. Nemery, F. Lauters
}

\section{To cite this version:}

G. Antoine, M. Jodeau, B. Camenen, M. Esteves, J. Nemery, et al.. Estimation des flux de matières en suspension lors des chasses hydrauliques de l'Arc de 2006 À 2011. La Houille Blanche - Revue internationale de l'eau, 2013, 4, pp.43-49. 10.1051/lhb/2013032 . hal-01118963

\section{HAL Id: hal-01118963 \\ https://hal.science/hal-01118963}

Submitted on 20 Feb 2015

HAL is a multi-disciplinary open access archive for the deposit and dissemination of scientific research documents, whether they are published or not. The documents may come from teaching and research institutions in France or abroad, or from public or private research centers.
L'archive ouverte pluridisciplinaire HAL, est destinée au dépôt et à la diffusion de documents scientifiques de niveau recherche, publiés ou non, émanant des établissements d'enseignement et de recherche français ou étrangers, des laboratoires publics ou privés. 


\title{
Estimation des flux de matières en suspension lors des chasses hydrauliques de l'Arc de 2006 À 2011
}

\author{
Germain ANTOINE ${ }^{123}$, Magali JODEAU ${ }^{1}$, Benoît CAMENEN² ${ }^{2}$, Michel ESTEVES 3 , \\ Julien NEMERY ${ }^{4}$, François LAUTERS ${ }^{5}$
}

\author{
1.EDF - R\&D - LNHE - 6 quai Watier, 78401 Chatou Cedex, France - germain.antoine@edf.fr, magali.jodeau@edf.fr \\ 2. Irstea - UR HH - 5 rue de la Doua, CS7007769626 Villeurbanne Cedex- benoit.camenen@irstea.fr \\ 3.Université Grenoble Alpes, CNRS, IRD, LTHE, F-38000 Grenoble,France-michel.esteves@ird.fr \\ 4.Université Grenoble Alpes, CNRS, IRD, LTHE, F-38000 Grenoble, France-julien.nemery@grenoble-inp.fr \\ 5.EDF-DTG - 21, avenue de l'Europe B.P.4138040 Grenoble Cedex09 - francois.lauters@edf.fr
}

\begin{abstract}
RÉSUMÉ. - En rivière aménagée de montagne, l'optimisation du fonctionnement des ouvrages hydro-éléctriques et de leur impact sur le cours d'eau passe par une meilleure compréhension des flux de matières en suspension (MES). Sur l'Arc en Maurienne, des chasses hydrauliques des trois principales retenues EDF sont réalisées tous les ans. Durant ces épisodes de chasse, les sédiments accumulés dans les retenues sont érodés et transportés en aval des ouvrages. Pour étudier la dynamique de ce transport, 5 campagnes de mesures ont été réalisées pendant les chasses de juin 2006, 2007, 2009, 2010 et 2011. Des mesures de débits et de concentrations en MES ont été réalisées en plusieurs sites de mesures entre le barrage de Saint-Martin-La-Porte et la ville de Grenoble. Pour compléter ces données, un modèle hydraulique 1D a été réalisé (code MASCARET) sur l'ensemble du domaine d'étude, pour chacune des chasses. En 14 sites de mesures, des flux massiques de MES ont ainsi été calculés pour ces chasses hydrauliques. Un modèle de propagation de l'incertitude est proposé pour prendre en compte les principales sources d'erreur intervenant dans le calcul du flux global : méthode de mesure, modélisation hydraulique et hétérogénéité de la concentration en MES dans une section en travers. Il apparaît que la dynamique d'échange de MES avec le lit de la rivière et la quantité de sédiments remobilisés des retenues peuvent être très différents d'une année à l'autre, et les valeurs d'incertitude confirment les tendances globales de flux observées le long du système Arc-Isère. Néanmoins, les bilans de masses d'un site à l'autre apparaissent plus délicats à interpréter.
\end{abstract}

Mots-clés : rivière alpine, MES, chasse, érosion, barrage

\section{Assessment of suspended sediment fluxes during Arc flushing flows, 2006-2011}

\begin{abstract}
Dam flushing is conducted regularly on the Arc River (Northern French Alps) to remove sediments from dam reservoirs. This paper presents the analysis of five field campaigns carried out during dam flushing events in the upper Arc River basin (in June 2006, 2007, 2009, 2010 and 2011). Measurements of suspended sediment concentrations (SSC) and discharges were performed at several sampling sites along the river channel. To complete the discharge data set, a 1D numerical hydraulic model, MASCARET, was calibrated and used to estimate the instantaneous discharge at any position along the river system. Following the temporal integration of the instantaneous suspended sediment fluxes, a spatial distribution of the suspended load along the Arc and Isère Rivers was obtained. The classical error propagation method was used to quantify errors resulting from the method employed to estimate the integrated fluxes, by taking into account the relative errors introduced by both the measurements and the model. It appears that the response of the river's sediment transport differs from one year to another. The quantity of fine sediments removed from the reservoirs varied from 10,000 tons in 2007 to 40,000 tons in 2006. Over the 120 kilometers of river network, the suspended load could either increase significantly (30,000 tons in 2007) or else remain exactly the same (in 2009 and 2011). The mean relative standard deviation on the integrated fluxes is equal to $11 \%$. Taking into account these uncertainty values, it appears that the global tendencies are significant, but the local mass balances are more difficult to interpret.
\end{abstract}

Key-words: alpine river, TSS,discharge, erosion, dam

\section{INTRODUCTION}

Les flux de sédiments en suspension représentent une part non négligeable des flux solides dans les cours d'eau. A l'échelle mondiale, le flux exporté vers les océans est estimé entre 15 et 20 milliards de tonnes en moyenne annuelle (Owens et al., 2005). Le flux annuel de matières en suspension (MES) des fleuves français est de l'ordre de 19 millions de tonnes (Delmas, 2009). La contribution des cours d'eau de montagne est considérée dans la littérature comme prédominante (Milliman \& Syvitski, 1992). Par exemple en France, le Rhône et la Loire contribuent respectivement à $52 \%$ et $19 \%$ au bilan national (Delmas, 2009), pour des surfaces de bassins versant respectives de $84.10^{3}$ et $115.10^{3}$ $\mathrm{km}^{2}$ (respectivement $12 \%$ et $17 \%$ de la superficie totale du territoire français).

Même si leur rôle sur la morphologie des rivières est moins marqué que celui des sédiments grossiers charriés, 
les sédiments fins ont une influence sur la morphologie des cours d'eau, notamment par la fixation de la végétation et l'exhaussement des bancs (Allain Jegou, 2002 ; Asaeda \& Rashid, 2012). Ils contribuent également de façon dominante à la morphologie des estuaires : à titre d'exemple, leur insuffisance renforce la vulnérabilité du delta de l'Ebre (Fatoric \& Chelledri, 2012). Par ailleurs, le milieu aquatique peut être fortement impacté par des modifications de la qualité ou de la quantité de sédiments fins. Des concentrations en MES élevées affectent les dynamique de population des macro-invertébrés (Jones et al., 2012 ; Crosa et al., 2010) et des poissons (Bilotta et al., 2012). Enfin, les MES jouent un rôle sur le transport des polluants, par fixation des polluants d'origine agricole, urbaine ou industrielle (Coynel et al, 2007).

Ces flux de MES sont susceptibles d'être modifiés par l'activité anthropique ou les évolutions climatiques : l'exploitation des sols modifie les taux d'érosion, les retenues de barrage sont susceptibles de stocker tout ou une partie des sédiments. Le fonctionnement des aménagements hydroélectriques peut être altéré par la présence des sédiments (accumulations devant des vannes, usure des turbines...). Pour permettre d'estimer les impacts des flux sédimentaires, de leur évolution dans un contexte de changement climatique ou d'évolution des usages de l'eau et des sols, il est donc nécessaire de disposer de moyens fiables d'estimation des flux de MES et des incertitudes associées.

On se propose d'illustrer la démarche d'estimation des flux de MES et des incertitudes associées par un exemple : l'Arc en Maurienne. Sur cette rivière, pour laquelle le flux annuel de MES représente environ $70 \%$ du débit solide total (Marnézy, 1998), des estimations de flux de MES sont proposées pour l'aval des aménagements hydroélectriques d'EDF, lors des chasses de barrages entre 2006 et 2011. Dans un premier temps les méthodes de mesure utilisées sont présentées, puis l'estimation des incertitudes est détaillée, enfin le calcul de flux sédimentaire est effectué pour les chasses de l'Arc de 2006 à 2011 et les résultats sont discutés.

\section{MÉTHODES DE MESURE ET ESTIMATION DES FLUX EN SUSPENSION}

\section{II.1. Description du site Arc-Isère}

Le bassin versant de 1'Isère à Grenoble $\left(5570 \mathrm{~km}^{2}\right)$ se situe dans le Sud-Est de la France (Alpes françaises) (Figure 1). C'est un bassin typique de montagne avec $77 \%$ du bassin au-dessus de $1000 \mathrm{~m}$ d'altitude, un régime hydrologique de type pluvio-nival (précipitations annuelles moyennes de $800 \mathrm{~mm}$, débit moyen annuel de $200 \mathrm{~m}^{3} / \mathrm{s}$ ) et caractérisé par un fort transport en sédiment en suspension (Dumas 2007, Némery et al, 2012). Ce bassin est fortement aménagé pour la production d'hydroélectricité en particulier sur son affluent amont de l'Arc (Lauters et al, 2012). Ce bassin versant (surface $1950 \mathrm{~km}^{2}$ ) compte 3 barrages au fil de l'eau (Saint Martin la Porte, Pont des Chèvres et Freney sur l'Arc (Fig. 1) dans la vallée de la Maurienne. Ces barrages sont chassés simultanément chaque année au mois de juin. Les vannes des trois barrages sont alors ouvertes progressivement pour produire un hydrogramme de chasse, caractérisé par une montée rapide des eaux et le maintien successif pendant environ 8 heures de deux paliers de débits (respectivement de $70 \mathrm{~m}^{3} / \mathrm{s}$ pendant 3-4 heures, puis de $120 \mathrm{~m}^{3} / \mathrm{s}$ pendant $4-5$ heures), avant de revenir au débit initial (Camenen et al., 2008). En cas de crue significative dans les mois précédents, les barrages sont rendus transparents au cours de l'événement et la chasse hydraulique annuelle est annulée. Cette situation s'est produite en 2008.

Durant les cinq chasses de l'Arc entre 2006 et 2011, des campagnes de mesures ont été menées le long de l'Arc et de l'Isère, depuis l'aval du barrage de Saint-Martin-La-Porte jusqu'à la ville de Grenoble. Sur cette distance, 14 sites de mesures ont été choisis.. La figure 1 présente l'ensemble de ces 14 sites de mesures. Par la suite, la position des sites de mesures sera définie par la distance du site de mesure à la confluence Arc-Isère (ces distances, notées pK et exprimées en kilomètres, seront donc négatives sur l'Arc et positive sur l'Isère).

Sur les 14 sites de mesures, des mesures de concentrations en MES ont été réalisées pendant les chasses. Sur quatre de ces sites, des débits ont également été mesurés. Enfin, des mesures complémentaires en débit et MES ont été réalisées au droit des principaux affluents. Pour chacune des chasses considérées dans ce travail, la contribution des affluents et de l'Isère amont en terme de flux de MES s'est révélée négligeable et ne sera donc pas prise en compte.

\section{II.2. Mesure et estimations des débits}

Pour la construction des courbes de tarage, a plupart des mesures de débits se font via la méthode d'exploration des champs de vitesse (Perret, 2009). Cette méthode est particulièrement fiable lorsque la section en travers se situe au droit d'un ouvrage de génie civil de géométrie stable et connue, ou dans une partie peu dynamique de la rivière. Elle a été utilisée sur les sites de mesure I1, I3, A5 et A7 (fig. 1). En outre, il peut être délicat de réaliser ces mesures pour des fortes valeurs de débits, par exemple pendant des épisodes de crues. Dans ce cas, des méthodes innovantes et non intrusives peuvent être utilisées pour estimer le débit, comme par exemple des mesures de vitesse de surface par LSPIV (Large Scale Particle Image Velocimetry). De telles mesures ont été réalisées pour compléter la courbe de tarage de la station de mesure A7 sur l'Arc en Maurienne (Dramais et al., 2011).

Dans cette étude, pour avoir une estimation des débits entre deux stations fixes de mesures, un modèle hydraulique unidimensionnel de l'Arc et de l'Isère a été établi avec le code de calcul MASCARET (Goutal \& Maurel, 1996), partie intégrante du système open-source TELEMAC-MASCARET. Près de 300 profils en travers, levés pendant plusieurs campagnes de mesures topographiques, ont été utilisés pour construire la géométrie du modèle le long des 120 kilomètres du système Arc-Isère. Les hydrogrammes de chasses mesurés au site de mesure A7 ont été translatés dans le temps et utilisés comme condition limite amont du modèle à l'aval du barrage de Saint Martin La Porte (1 kilomètre en amont du premier site de mesure A10). La condition limite aval est donnée par la loi hauteur-débit mesurée à la station hydrométrique de Grenoble Campus. Les débits d'apports ont été définis aux trois principaux affluents du bief considéré : au niveau de la restitution de l'usine de La Coche-Randens (10 km en amont de la confluence Arc-Isère), au niveau de la confluence Arc-Isère, et au niveau de la restitution du bassin du Cheylas (environ $30 \mathrm{~km}$ en aval de la confluence Arc-Isère). Le modèle a été calé via les valeurs de coefficient de frottement à partir des hauteurs d'eau mesurées aux quatre sites de mesure de débit décrits plus haut, ainsi que sur les temps de propagation du pic de débit de l'hydrogramme de chasse. Les valeurs de coefficients de Strickler, croissantes le long du linéaire, sont cohérentes avec la granulométrie observée sur le lit. Les débits calculés ont ainsi 


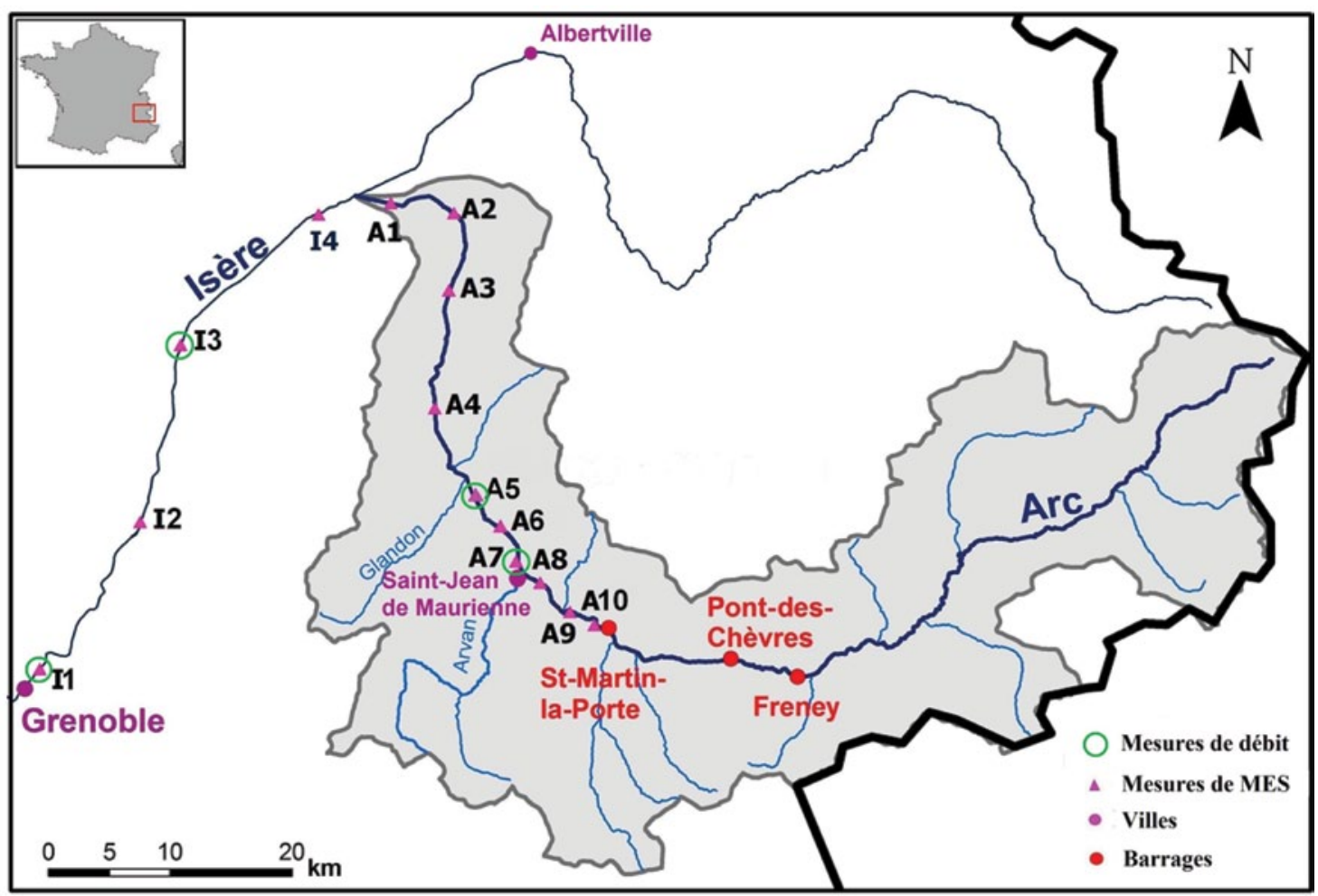

Figure 1 : Localisation du barrage de Saint Martin la Porte et des 14 sites de mesure des concentrations de MES (triangles violets) et de débit (cercles verts) sur l'Arc et l'Isère.

été contrôlés aux 4 stations de mesures hydrométriques, et les écarts relatifs moyens sur chaque hydrogramme sont en majorité inférieurs à $5 \%$, et jamais supérieur à $10 \%$ (l'erreur la plus forte se faisant sur les plus bas débits, peu contributeurs dans le calcul du flux de MES).

\section{II.3. Mesure et estimation des concentrations en MES}

La méthode de mesure de concentration en MES la plus utilisée sur les sites de mesures cités précédemment consiste dans un premier temps à effectuer un prélèvement au sein de la colonne d'eau. La concentration en matière en suspension est obtenue par filtration/pesée (norme ISO 11923). Plusieurs stratégies de prélèvement existent. Selon la configuration du site de mesure, il peut être choisi d'effectuer des prélèvements au seau depuis un pont. Le prélèvement se fait alors en surface. Le contenu du seau est ensuite ré-échantillonné pour être versé dans un flacon, qui sera enfin analysé en laboratoire. Un préleveur automatique peut aussi être installé sur le site de mesure, afin de s'affranchir de la lourdeur de prélèvements manuels répétés. Une crépine d'aspiration est alors installée dans la colonne d'eau, et une pompe prélève de façon automatique sur une période de 30 secondes des échantillons normés en volume qui rempliront directement des flacons à analyser en laboratoire, supprimant ainsi une étape de ré-échantillonnage et moyennant sur 30 secondes la valeur de concentration. La crépine d'aspiration est fixée de telle façon qu'elle ne soit jamais hors d'eau le temps de la mesure. Ces deux méthodes de prélèvement ont été utilisées sur les 14 sites de mesure, avec au fil des ans une systématisation de l'utilisation d'un préleveur automatique. A noter que les deux méthodes ont été comparées, et l'utilisation d'un préleveur a surtout l'avantage de pouvoir augmenter à moindre coût la fréquence d'échantillonnage, le biais sur la valeur de concentration étant inférieur à $3 \%$.

En complément, des turbidimètres (Hach Lange Solitax SC-Line TS $50 \mathrm{~g} / \mathrm{L}$ ) ont été utilisés sur certains sites de mesures (A7, A5, I3 et I1). La mesure de concentration en MES par turbidimétrie consiste à plonger un capteur optique dans l'écoulement, qui va mesurer la turbidité locale de l'eau (au voisinage de la sonde) par rétrodiffusion. En étalonnant ces mesures par des prélèvements réalisés aux mêmes instants, on construit une relation entre la concentration en MES et la turbidité mesurée. Cette relation, une fois extrapolée, permet de convertir les données de turbidités mesurées en continu en concentration en MES. La fréquence d'échantillonnage, de l'ordre de une mesure moyenne toutes les 30 secondes, est alors bien plus élevée qu'avec les méthodes décrites précédemment, mais la relation entre la concentration en MES et la turbidité introduit un biais, quantifié dans la partie suivante.

\section{II.4. Estimation des flux de MES et des incertitudes associées}

Le flux instantané $\varphi(t)$ (exprimé en $[\mathrm{kg} / \mathrm{s}]$ ) est obtenu par simple produit du débit liquide $Q(t)\left(\mathrm{en}\left[\mathrm{m}^{3} / \mathrm{s}\right]\right)$ et de la concentration massique $C(t)$ (en $\left.\left[\mathrm{kg} / \mathrm{m}^{3}\right]\right)$ au même instant t:

$$
\varphi(t)=Q(t) \times C(t) .
$$

Il convient ensuite de l'intégrer sur une période $\mathrm{T}$ choisie pour obtenir le flux total $\Phi$ transité sur cette période :

$$
\Phi=\int_{T} \varphi(t) d t
$$


Nous proposons ici un modèle simple de propagation de l'incertitude, afin de prendre en compte les différentes sources d'incertitudes qui entrent en jeu dans le calcul du flux de MES global.

Le flux instantané s'exprime comme le produit de la concentration en MES et du débit d'eau instantané ; ces deux valeurs provenant de mesures indépendantes, la variance relative du flux instantané peut s'exprimer comme la somme des variances relatives liées au débit et à la concentration en MES :

$$
\sigma_{\varphi}^{2}=\sigma_{Q}^{2}+\sigma_{C}^{2}
$$

Conformément à la méthodologie développée dans le guide pour l'expression de l'incertitude de mesure (GUM en anglais (JGCM, 2008)), il convient ensuite de propager l'incertitude en cascade pour chacune des variables, en prenant en compte les principales sources d'incertitudes. Notons ici que nous considérerons dans cette étude une unique valeur de variance par site de mesure, pour toute la durée de l'évènement. Cette hypothèse est une simplification justifiée dès lors que seules les valeurs les plus élevées de débit et concentration en MES sont réellement contributrices du flux total de MES. La valeur $\sigma_{\varphi}^{2}$ liée au flux instantané devient ainsi indépendante du temps, et pourra être assimilée à la variance relative du flux intégré $\sigma_{\Phi}^{2}$ sur toute la période de l'évènement.

\section{II.4.1. Incertitudes liées aux valeurs de débits}

Dans cette étude l'incertitude liée aux valeurs de débit peut provenir de trois sources principales : la mesure, la modélisation numérique et l'interpolation en temps. La variance de la valeur de débit peut donc s'exprimer comme la somme des trois variances liées à ces sources d'incertitudes :

$$
\sigma_{Q}^{2}=\sigma_{Q-M e s}^{2}+\sigma_{Q-M o d}^{2}+\sigma_{Q-I n t}^{2}
$$

Le Coz et al. (2012) estiment une incertitude liée à la mesure de débit associée à une courbe de tarage de l'odre de $7 \%$ au site de mesure A2. Cette estimation résulte de l'analyse de résultats obtenus pendant une campagne de mesure de 2011. Les auteurs montrent dans ce travail que ces valeurs d'incertitude sont spécifiques au site d'étude. Cette valeur étant la plus précise établie sur le réseau Arc-Isère, nous l'appliquerons néanmoins pour tous les sites de mesures de débit (approximée à $\sigma_{Q-M e s}=7 \%$ ), mais aussi sur les sites ne disposant pas de mesures de débit, car cette incertitude se cumule à celle liée à l'étape de modélisation. Cette valeur confirme par ailleurs l'ordre de grandeur estimé par Olivier et al. (2008). Pour les sites ne disposant pas de mesures de débit, et uniquement pour ceux-ci, s'ajoute la valeur $\sigma_{Q-M o d}$ liée au modèle. En chaque site de mesure de débit et pour chaque année, l'écart type moyen entre les valeurs mesurées et les valeurs calculées par le modèle est estimé. Ces valeurs varient entre $2,9 \%$ et $7,4 \%$ en fonction du site de mesure considéré et de l'année. Pour calculer $\sigma_{Q-M o d}$, une interpolation linéaire spatiale est ensuite appliquée aux sites de mesures de concentration se situant entre deux sites de mesure de débits consécutifs (avec une valeur nulle aux sites de mesure). Enfin, les valeurs de débits étant disponibles de façon quasi-continue (par le modèle ou la mesure), le terme $\sigma_{Q-\text { Int }}$ lié à l'interpolation linéaire temporelle sera négligé.

\section{II.4.2. Incertitudes liées aux valeurs de concentration en MES}

L'incertitude liée aux valeurs de concentrations en MES peut provenir de quatre sources principales : la méthode de mesure, l'hétérogénéité spatiale de la concentration dans la section en travers de la rivière (deux composantes horizontale et verticale) et l'interpolation temporelle. La variance de la valeur de concentration en MES peut donc s'exprimer comme la somme des quatre variances liées à ces sources d'incertitudes :

$$
\sigma_{C}^{2}=\sigma_{C-M e s}^{2}+\sigma_{C-H H}^{2}+\sigma_{C-H V}^{2}+\sigma_{C-I n t}^{2}
$$

L'incertitude provenant de la méthode de mesure prendra deux valeurs différentes, en fonction de la méthode utilisée : prélèvement (automatique ou manuel) ou utilisation d'un turbidimètre. Gautheron (1994) a réalisé des tests de répétabilité pour estimer l'incertitude liée au cycle filtration-séchage-pesée. Sur la base de cette étude, et au regard des concentrations en MES mesurées sur l'Arc et l'Isère pendant les chasses, nous considérerons que $\sigma_{C-M e s}=5 \%$ pour les prélèvements. Pour les sites sur lesquels un turbidimètre a été utilisé, Navratil et al. (2011) ont montré que pour des concentrations comprises entre $1 \mathrm{~g} / \mathrm{l}$ et $10 \mathrm{~g} / \mathrm{l}$, l'écart type à $95 \%$ de degré de confiance associé à l'utilisation d'un turbidimètre en rivière de montagne s'élève à $10 \%$ (cette valeur décroît ensuite pour des concentrations plus élevées). Les turbidimètres utilisés durant les campagnes de mesures sur l'Arc ont mesuré des concentrations souvent comprises dans cette gamme. Nous considérerons donc dans cette étude que $\sigma_{C-M e s}=5 \%$ pour les sites équipés d'un turbidimètre.

Pour estimer l'incertitude liée à l'hétérogénéité horizontale dans une section en travers, des mesures de surface le long d'une section en travers ont été réalisées par Mano (2008) pendant la chasse de 2006 en I1. La valeur issue de ces mesures est de $\sigma_{C-H H}=5 \%$, que nous appliquerons sur l'ensemble des sites. Du fait des fortes vitesses de l'écoulement, aucune mesure de prélèvement sur la verticale n'a été faite pendant ces chasses. Néanmoins, des mesures de vitesses de chute des MES réalisées en 2011 (Antoine et al., 2012), couplées à des valeurs de vitesses de frottement de fond calculées par le modèle hydraulique nous permettent d'avoir une estimation du nombre de Schmidt-Rouse. Ce dernier donne la possibilité d'estimer un profil vertical théorique, et donc un écart type relatif à la valeur moyenne le long de ce profil vertical. Une valeur représentative calculée par cette méthode sur l'ensemble des sites de mesure est $\sigma_{C-H V}=3 \%$.

Enfin, l'erreur liée à l'échantillonnage sera estimée en chaque site de mesure et pour chaque chasse, par le rapport moyen entre les grandeurs $\left|C_{i+1}-C_{i}\right| \times\left(t_{i+1}-t_{i}\right)$ et $T_{\text {chasse }} \times\left(C_{i}+C_{i+1}\right) / 2$. Les valeurs ainsi calculées varient de $0.004 \%$ à $24 \%$ en fonction de la fréquence d'échantillonnage (qui varie elle-même entre une et 120 mesures par heure).

\section{FLUX GLOBAL ET INCERTITUDE : RESULTATS}

Les valeurs maximales de débit mesurées pendant les chasses varient de 120 à $160 \mathrm{~m}^{3} / \mathrm{s}$ sur l'Arc, et de 200 à $400 \mathrm{~m}^{3} / \mathrm{s}$ à Grenoble. Les concentrations maximales en MES mesurées varient selon l'épisode de chasse considéré de 
$10 \mathrm{~g} / \mathrm{l}$ à $30 \mathrm{~g} / \mathrm{l}$ sur l'Arc, et de $4 \mathrm{~g} / \mathrm{l}$ à $8 \mathrm{~g} / \mathrm{l}$ à Grenoble. La figure 2 représente la distribution spatiale des flux intégrés sur toute la période de chasse (variant de 12 à 16 heures en fonction du site à cause de la dispersion), pour les 5 chasses considérées en chaque site de mesure.

On constate sur cette figure que les flux de MES totaux en sortie de la retenue de Saint Martin la Porte (premier point de mesure, abscisse $\mathrm{pK}=-50 \mathrm{~km}$ ) varient entre 10000 et 40000 tonnes selon l'année considérée, et que la dynamique d'échange avec le lit de la rivière diffère également en fonction des années. Par exemple, en 2009 et 2011, une quasi conservation du flux est observée sur l'ensemble du bief (égalité du flux calculé au premier site et celui calculé au dernier), alors qu'en 2007 une augmentation nette du flux (de 30000 tonnes) est observée sur l'Arc en amont de la confluence avec l'Isère. Ainsi, pour un même hydrogramme théorique servant de base pour chacune de ces chasses, la quantité de sédiments remobilisés dans les retenues peut être multipliée par quatre d'une année sur l'autre, et la dynamique d'échange avec le lit de la rivière peut être fondamentalement différente. Cette différence peut s'expliquer par l'effet du passé hydrologique proche précédant la chasse, mais également par la différence de débits de base de l'Isère accueillant l'hydrogramme de chasse. Par exemple, le faible débit de base de l'Isère en 2011 pourrait être responsable du phénomène de dépôt observé juste en aval de la confluence Arc-Isère.

Car si la tendance globale est à l'érosion sur l'ensemble du bief (bilan de masse positif ou nul entre le premier et le dernier site de mesure), ces courbes montrent également de fortes variations locales négatives. Il est donc nécessaire d'analyser ces courbes de flux au regard de l'incertitude liée à cette valeur de flux (tableau 1).

Les calculs indiquent des incertitudes sur le flux de MES de l'ordre de $12 \%$. Plus précisément, les valeurs d'écart type relatif varient entre $10.4 \%$ et $26.2 \%$, avec une valeur moyenne de $11.8 \%$ et une valeur médiane de $11.6 \%$. La dispersion de ces valeurs d'écart type relatif n'est donc pas très importante, et les grandes valeurs d'incertitudes résultent principalement d'un trop faible échantillonnage des concentrations en MES. Il n'en reste pas moins que suivant les méthodes de mesures de la concentration en MES et l'utilisation ou non d'un modèle numérique pour les valeurs de débits, cette incertitude est variable dans l'espace et dans le temps. On remarque une amélioration nette des valeurs d'écart type à partir de 2007, confirmée après 2009, grâce à l'utilisation systématique de préleveurs automatiques plutôt que des prélèvements au seau. Pour une même année, on remarque également l'impact de l'utilisation (ou non) d'un modèle numérique sur les valeurs d'incertitude.

Le poids relatif de l'incertitude sur la mesure de concentration par rapport à l'incertitude sur le débit (Figure 3a) est également variable dans l'espace, tout en restant la plupart du temps du même ordre de grandeur. Ces conclusions ne sont valables que pour les différentes valeurs d'incertitudes choisies. La méthode, plus générale, pourrait être adaptée sur un autre site ou modifiée avec de nouvelles valeurs d'écart type mesurées. Quoiqu'il en soit, l'estimation de ces incertitudes permet d'identifier les sites de mesures pour lesquels les variations spatiales du flux ne seraient pas significatives.

Par exemple, la figure $3 \mathrm{~b}$ montre les intervalles de confiance à $95 \%$ (2 écarts type) et $70 \%$ (1 écart type) résultant du calcul d'incertitude sur le flux en 2010 : on constate que si la tendance globale à l'érosion est vérifiée (remise en suspension de 20000 tonnes de MES entre le $9^{\text {ème }}$ et le $10^{\text {ème }}$ site de mesure), la plupart des variations locales ne sont pas significatives. Cette tendance est confirmée pour l'ensemble des chasses étudiées. Il semble donc, que l'incertitude se soit stabilisée autour d'une valeur d'environ $11 \%$ (voir 2011 dans le tableau 1). Cette valeur ne permet pas de confirmer les faibles variations de flux d'un site de mesure à l'autre. Malgré tout, les variations spatiales de flux sur l'ensemble du domaine sont souvent le résultat de fortes variations locales, et en ce sens, la densification du réseau n'était pas vide de sens. Il conviendrait à présent de limiter les sites de mesure à des bornes de tronçons de rivière homogènes, qu'un modèle numérique ou d'observations de terrain plus précises permettraient de définir.

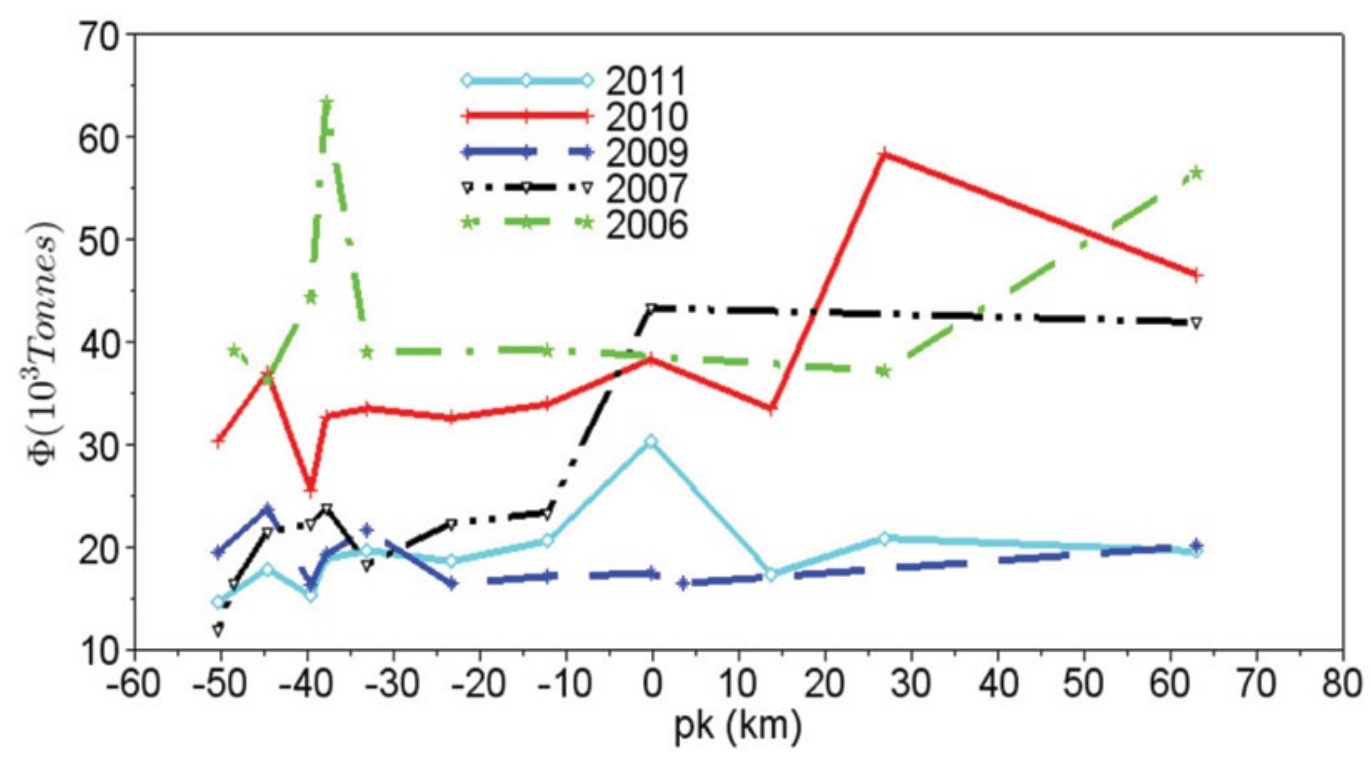

Figure 2 : Flux sédimentaires calculés entre 2006 et 2011 sur l'Arc et l'Isère (confluence Arc-Isère à pK =0 km). 
Tableau 1 : Valeurs de flux total en chaque site de mesure $\Phi$ (exprimé en $10^{3}$ tonnes), et leur écart type relatif $\sigma_{\Phi}$ associé (exprimée en \%)

\begin{tabular}{|l|c|c|c|c|c|c|c|c|c|c|}
\hline $\begin{array}{l}\text { Site de } \\
\text { mesure }\end{array}$ & \multicolumn{2}{|c|}{2006} & \multicolumn{2}{c|}{2007} & \multicolumn{2}{c|}{2010} & \multicolumn{2}{c|}{2011} \\
\hline & $\Phi$ & $\sigma_{\Phi}$ & $\Phi$ & $\sigma_{\Phi}$ & $\Phi$ & $\sigma_{\Phi}$ & $\Phi$ & $\sigma_{\Phi}$ & $\Phi$ & $\sigma_{\Phi}$ \\
\hline A10 & - & - & 12.2 & 11.6 & 19.6 & 11.7 & 30.4 & 11.2 & 14.8 & 11.9 \\
\hline A9 & 39.3 & 13.5 & 16.6 & 11.7 & - & - & - & - & - & - \\
\hline A8 & 36.4 & 13.4 & 21.7 & 11.6 & 23.8 & 12 & 37.1 & 11.2 & 18 & 12 \\
\hline A7 & 44.5 & 12.4 & 22.3 & 10.5 & 16.5 & 10.4 & 25.6 & 10.4 & 15.3 & 10.4 \\
\hline A6 & 63.4 & 26.2 & 24 & 11.6 & 19.4 & 11.5 & 32.8 & 11.4 & 19 & 11.9 \\
\hline A5 & 39.1 & 10.5 & 18.5 & 10.4 & 21.7 & 10.5 & 33.6 & 10.4 & 19.8 & 10.5 \\
\hline A4 & - & - & 22.4 & 11.7 & 16.5 & 11.7 & 32.7 & 11.1 & 18.7 & 12.2 \\
\hline A3 & 39.3 & 13.1 & 23.5 & 11.8 & 17.3 & 11.8 & 34 & 11.2 & 20.7 & 12.3 \\
\hline A2 & - & - & - & - & - & - & - & - & - & - \\
\hline A1 & - & - & 43.4 & 16 & 17.5 & 12.1 & 38.4 & 11.2 & 30.4 & 12.5 \\
\hline I4 & - & - & 16.6 & 12.2 & - & - & - & - & - & - \\
\hline I3 & - & - & - & - & - & - & 33.6 & 10.5 & 17.5 & 10.5 \\
\hline I2 & 37.3 & 11.4 & - & - & - & - & 58.4 & 11.1 & 21 & 12.1 \\
\hline I1 & 56.6 & 10.9 & 42 & 20.2 & 20.2 & 10.4 & 46.6 & 10.4 & 19.7 & 10.4 \\
\hline
\end{tabular}

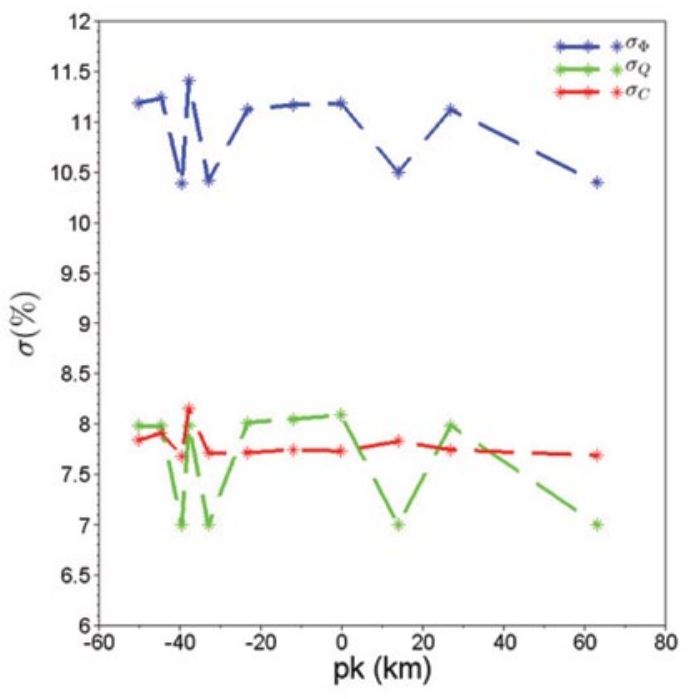

(a)

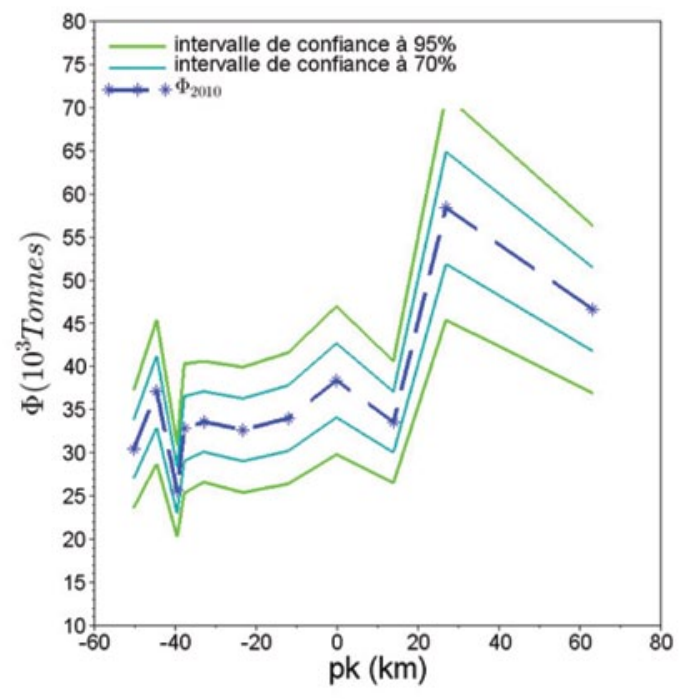

(b)

Figure 3 : Exemple de résultats d'incertitude pour la chasse de 2010 : (a) Décomposition de l'incertitude totale $\left(\sigma_{\Phi}\right)$ en incertitude sur le débit $\left(\sigma_{Q}\right)$ et incertitude sur la concentration $\left(\sigma_{C}\right)$; (b) Evolution spatiale du flux sédimentaires et intervalles de confiance à $95 \%$ et $70 \%$ associées.

\section{CONCLUSIONS \& PERSPECTIVES}

Dans cette étude, cinq campagnes de mesure réalisées pendant des chasses de barrage sur l'Arc en Maurienne ont été analysées : à partir des données de débits mesurées et complétées par un modèle numérique hydraulique 1D et des données mesurées de concentrations en MES, une distribution spatiale des flux de MES a été établie. Cette distribution spatiale montre de fortes hétérogénéités en terme de réponse sédimentaire aux évènements de chasse de barrage : d'une part, les quantités de sédiments remises en suspensions dans les retenues varient fortement d'une années sur l'autre (de 10000 tonnes en 2007 à 40000 tonnes en 2006), et d'autre part le bilan sédimentaire sur l'ensemble du domaine aval peut aussi bien être nul (comme en 2009), que positif (comme en 2007, où 30000 tonnes des sédiments ont été remis en suspension le long de la rivière). Un modèle d'incertitude a été appliqué au calcul de flux, permettant d'estimer une valeur d'incertitude moyenne de $12 \%$ sur ces calculs de flux. Dans ce calcul, la contribution de l'incertitude faite sur les mesures de concentrations en MES est du même ordre de grandeur que celle faite sur les mesures 
de débits. Ces valeurs d'incertitudes permettent ainsi de confirmer les tendances globales à l'érosion le long du lit des deux rivières. A l'inverse, peu de variations locales sont confirmées au regard des valeurs d'incertitudes. Ces résultats renforcent l'idée que pour progresser vers une compréhension fine de la dynamique spatiale et temporelle des flux de MES en rivières, des méthodes innovantes de mesures permettant une meilleure exploration des concentrations en MES au sein d'une section en travers (telles que les mesures acoustiques Moore, 2011) doivent être développées, et que l'utilisation d'un modèle numérique hydro-sédimentaire à l'échelle de la rivière permettrait de comprendre le comportement de façon plus intégré.

\section{REMERCIEMENTS}

Les auteurs souhaitent remercier les équipes de l'IRSTEA, d'EDF (DTG et LNHE), du LTHE qui ont participé aux nombreuses campagnes de mesure. Les mesures ont été réalisées sur le site atelier Arc-Isère labellisé auprès de la ZABR (Zone Atelier Bassin Rhône).

\section{RÉFERENCES}

Antoine G., Jodeau M., Camenen B. \& Esteves M. (2012) - A settling velocity parameterization for sand/mud mixture in a 1D flow during a flushing event. In River Flow, San Jose, Costa Rica. $1343 \mathrm{p}$

Asaeda T. \& Rashid M. H. (2012) - The impacts of sediment released from dams on downstream sediment bar vegetation. Journal of Hydrology. 430-431 25-38

Allain Jegou C. (2002) - Thèse Institut National Polytechnique de Grenoble. Relations végétation-écoulement-transport solide dans le lit des rivières, Etude de l'Isère dans le Grésivaudan. $206 \mathrm{p}$

Bilotta G.S., Burnside N.G., Cheek L., Dunbar M.J., Grove M.K., Harrison C., Joyce C. , Peacock C. \& Davy-Bowker J. (2012) - Developing environment-specific water quality guidelines for suspended particulate matter. Water Research. 46 2324-2332

Camenen B., Jodeau M. \& Le Coz J. (2008) - Modélisation du flux sédimentaire pendant une chasse hydraulique (Arc en Maurienne). La Houille Blanche. 4 83-89

Collier K. J. (2002) - Effects of flow regulation and sediment flushing on instream habitat and benthic invertebrates in a New Zealand river influenced by a volcanic eruption. River Res. Applic. 18 213-226

Coynel A., Schäfer J., Blanc G., Bossy C. (2007) - Scenario of particulate trace metal and metalloid transport during a major flood event inferred from transient geochemical signals. 2007. Applied Geochemistry. 22 821-836

G. Crosa, E. Castelli, G. Gentili, And P. Espa. (2010) - Effects of suspended sediments from reservoir flushing on fish and macroinvertebrates in an alpine stream. Aquatic Sciences. 72 85-95

Delmas M. (2009) - Origine des exports de sédiments fluviatiles : prise en compte de l'hétérogénéité spatiale des versants. Thèse Université Paris 1 Panthéon Sorbonne. 195p

Dramais G., Le Coz J., Camenen B., Hauet A. (2011) Advantages of a mobile LSPIV method for measuring flood discharges and improving stage discharge curves. Journal of Hydro-environment Research. 5 301-3012
Dumas D. (2007) - The results of 10 years of daily observations of the flux of suspended matter in one of the main watercourses in the European Alps: The Isere at Grenoble (France). Comptes Rendus de l'Académie des Sciences Geosciences. 339 810-819

FATORIC S. \& CHELledri L. (2012) - Vulnerability to the effects of climate change and adaptation: The case of the Spanish Ebro Delta. Ocean and Coastal Management. 60 1-10

GAUTHERON A. (1994) — Incertitudes sur les mesures des matières en suspension en Basse Durance. Rapport interne, EDF DTG

Goutal N. \& Maurel F. (2002) - A finite volume solver for 1D shallow-water equations applied to an actual river. International Journal for Numerical Methods in Fluids. 38 1-19

JCGM (2008) - Évaluation des données de mesure - Guide pour l'expression de l'incertitude de mesure. $132 \mathrm{p}$

Jones J. I.; Murphy J. F.; Collins A. L.; Sear D. A.; NADen P. S. \& ARmitage P. D. (2012) — The impact of fine sediment on macro-invertebrates. River Research and Applications. 28 1055-1071

Lauters F., Laperrousaz E., Camenen B., Le Coz J., Thollet F., Nemery J. \& Delinares M. (2010) - Vers une gestion sédimentaire durable de l'aménagement hydro-électrique Arc-Isère. La Houille Blanche. 1 p. 19-25

Le Coz J., Camenen B., Peyrard X. \& Dramais G. (2012) Uncertainty in open-channel discharges measured with the velocity area method. Flow Measurement and Instrumentation. 26 18-29

Mano V. (2008) - Processus conditionnant les apports de sédiments fins dans les retenues - optimisation des méthodes de mesure et modélisation. Thèse Institut National Polytechnique de Grenoble. $341 \mathrm{p}$

MARNEZY A. (1999) - L'Arc et sa vallée; anthropisation et géodynamique d'une rivière alpine dans son bassin versant. Thèse de doctorat à l'université Joseph Fourier Grenoble I. 667 p

Milliman J. \& SyvitSKi J. (1992) - Geomorphic/Tectonic Control of Sediment Discharge to the Ocean: The Importance of Small Mountainous Rivers. The Journal of Geology. 100. 525-5441

Moore S. A. (2011) - Monitoring flow and fluxes of suspended sediment in rivers using side-looking acoustic Doppler current profilers. Thèse Université de Grenoble. 220 p

Navratil O., Esteves M., Legout C., Gratiot N., Nemery J., Willmore S. \& Grangeon T. (2011) - Global uncertainty analysis of suspended sediment monitoring using turbidimeter in a small mountainous river catchment. Journal of Hydrology. 398 246-259

Némery J., Mano V., Coynel A., Etcheber H., Moatar F., Meybeck M., Belleudy P., Poirel A. (2012) - Carbon and suspended sediment transport in an impounded alpine river (Isere, France). DOI : 10.1002/hyp.9387. Hydrological Processes

Olivier A., Pierrefeu G., Scotti M. \& Blanquart B. (2008) Incertitude sur les débits calculés à partir des courbes de transformation hauteur/débit. Mesures hydrologiques et incertitudes. conférence SHF. Paris, France, 2008

Owens P., Batalla R., Collins A., Gomez B., Hicks D., Horowitz A., Kondolf G., Marden M., Page M., Peacock D., Petticrew E., Salomons W. \& Trustrum N. (2005) - Fine-grained sediment in river systems: environmental significance and management issues. River Research and Applications. 21 693-717

Perret C. (2009) — Les moyens de contrôle et de mesure des débits - les capteurs et les méthodes. La Houille Blanche. 3 97-107

Wren D., Barkdoll B., Kuhnle R. \& Derrow R. (2000) - Field techniques for suspended-sediment measurement. Journal of Hydraulic Engineering. 126 97-104 